\title{
Neugestaltung urbaner Freiräume - Einblicke in das Reallabor Wuppertal
}

\author{
Alexandra Palzkill, Karoline Augenstein \\ Eingegangen: 31. Juli 2020 - Angenommen: 22. März 2021 - Online veröffentlicht: 23. April 2021
}

\section{Zusammenfassung}

Städte als Orte der Nachhaltigkeitstransformationen erlangen immer größere Bedeutung in der Nachhaltigkeits- und Transformationsforschung. Eine der Schlüsselfragen ist, wie sich urbane Nachhaltigkeitsnischen proaktiv fördern und verbreiten lassen. Reallabore sind dabei ein Ansatz, um mithilfe von Experimenten an der Schnittstelle von Wissenschaft und Praxis Nachhaltigkeitsherausforderungen im konkreten (urbanen) Kontext zu reflektieren und komplexe Transformationsprozesse zu gestalten. Dieser Beitrag diskutiert Reallabore als reflexive Experimentier- und Lernräume und führt institutionelle Logiken und Narrative als konzeptionelle Zugänge zu deren Analyse ein. Dieser Ansatz wird anhand einer Fallstudie aus dem Reallabor Wuppertal illustriert. Gemeinsam mit der zivilgesellschaftlichen Initiative „Utopiastadt“ wurde ein Experiment zur Gestaltung lebenswerten urbanen Freiraums durchgeführt. Anhand der Ergebnisse der Fallstudie werden methodische Schlüsselmerkmale und das spezifische Verständnis von Experimenten in der Reallaborforschung diskutiert. Diese werden zu experimentellen Ansätzen in der Planungswissenschaft in Bezug gesetzt, um abschließend die jeweils unterschiedlichen Ausgangspunkte und Erwartungen bezüglich des Einsatzes von Experimenten sowie die potenzi-

Jun.-Prof. Dr. Alexandra Palzkill, Fakultät für Wirtschaftswissenschaft, Bergische Universität Wuppertal, Gaußstraße 20, 42119 Wuppertal, Deutschland

palzkill@uni-wuppertal.de

Jun.-Prof. Dr. Karoline Augenstein, Institut für Politikwissenschaft, Bergische Universität Wuppertal, Gaußstraße 20, 42119 Wuppertal, Deutschland

augenstein@uni-wuppertal.de

(c) (i)

(c) 2021 Palzkill; licensee oekom verlag. This Open Access article is published under a Creative Commons Attribution 4.0 International License. ellen Anknüpfungspunkte zwischen den beiden Forschungsbereichen zu diskutieren.

Schlüsselwörter: Urbane Reallabore - urbaner Freiraum • zivilgesellschaftliche Initiativen = Experiment - Reflexivität Wuppertal

\section{Re-designing and re-imagining livable urban space - Insights from the real-world lab Wuppertal}

\begin{abstract}
Cities as places of sustainability transformation are becoming increasingly important in sustainability and transition research. One of the key questions is how to proactively promote and spread urban sustainability niches. Real-world laboratories are an approach that uses experiments at the sciene-society interface to reflect sustainability challenges in a concrete (urban) context and to proactively foster complex transformation processes. This paper discusses real-world labs as spaces for experimentation and reflexive learning and introduces institutional logics and narratives as conceptual approaches to analyse these. This approach is illustrated using a case study from the real-world laboratory Wuppertal. Together with the civil society initiative "Utopiastadt", an experiment was carried out to develop liveable urban space. Based on the results of the case study, key methodological features and the specific understanding of experiments in real-world labs are discussed. They are related to experimental approaches in planning science in order to discuss the different understandings and expectations with regard to the use of experiments as well as the potential contributions and complementarities between the two fields of research.
\end{abstract}

Keywords: Urban real-world labs - Livable urban space Civil society initiatives $=$ Experiments - Reflexivity - Wuppertal 


\section{Einleitung: Lebenswerte Freiräume und die Rolle von Experimenten in urbanen Transformationen}

Die Forschung über gesellschaftlichen Wandel in Richtung Nachhaltigkeit nimmt vermehrt Städte als Orte der Nachhaltigkeitstransformation in den Fokus. Sie werden als Orte untersucht, in denen sich sowohl die Herausforderungen gesellschaftlicher Transformation zeigen, wo aber vor allem auch relevante Impulse für Veränderungen hin $\mathrm{zu}$ einer nachhaltigen Entwicklung initiiert und ausprobiert werden. Insbesondere in der Transition-Forschung hat sich ein Schwerpunkt zur Erforschung urbaner Transformationsprozesse entwickelt (Wolfram/Frantzeskaki 2016; Wolfram/Frantzeskaki/Maschmeyer 2016; Hodson/Geels/ McMeekin 2017). Während Fragen von Ort und Raum hier lange Zeit eher vernachlässigt wurden (Bulkeley/Castán Broto/Hodson et al. 2011; Hodson/Geels/McMeekin2017), werden inzwischen die räumlichen Kontexte von Städten zunehmend als relevant für die Erforschung und Schaffung von Potenzialen für Nachhaltigkeitstransformationen anerkannt. Es werden dabei Bezüge zur Stadtplanung und Raumforschung hergestellt, um die räumliche Heterogenität und lokalspezifische Interaktionen zwischen Akteuren und Strukturen zu beleuchten (Coenen/Benneworth/ Truffer 2012; Hansen/Coenen 2015; Raven/Sengers/Spaeth et al. 2019).

Das grundlegende Transformationsverständnis innerhalb der Transition-Forschung basiert unter anderem auf der
Multi-Level-Perspektive (Geels 2011), die den grundlegenden Wandel gesellschaftlicher (Sub-)Systeme als komplexen, koevolutionären Veränderungsprozess beschreibt, der zur Etablierung neuer institutioneller Regelsysteme, kultureller Normen und sozialer Praktiken führt (Grin/Rotmans/ Schot 2010: 11). Wie in Abbildung 1 dargestellt, vollzieht sich ein solcher Wandel komplexer Systeme über drei Ebenen hinweg: Nischen, Regime und landscape. Diese Ebenen beziehen sich explizit nicht auf räumliche Skalen, sondern unterscheiden sich durch unterschiedliche Grade von Strukturation (Grin/Rotmans/Schot 2010: 131; vgl. Giddens 1984). Damit basiert die Multi-Level-Perspektive auf der theoretischen Grundannahme, dass Akteure in ihrem Handeln von Strukturen geprägt sind, die sie selbst durch ihr Handeln (re)produzieren, wobei die Akteure grundsätzlich in der Lage sind, ihr Handeln kritisch zu reflektieren und zu verändern (vgl. Giddens 1984). In der Multi-Level-Perspektive beschreibt die Regimeebene das stark strukturierte institutionelle Gerüst und die dominanten Funktionslogiken eines Systems. Ein Regime produziert so einerseits Stabilität, gleichzeitig aber auch Pfadabhängigkeiten und Lock-Ins (Unruh 2000). Die Nischenebene ist im Vergleich dazu weniger stark strukturiert, hier werden proaktiv Handlungsspielräume geschaffen für die Entstehung von Neuerungen und Innovationen in geschützten Räumen. Die Landscape-Ebene mit dem höchsten Grad der Strukturation umfasst externe Einflussfaktoren, wie beispielsweise globale Entwicklungs- oder Megatrends, die

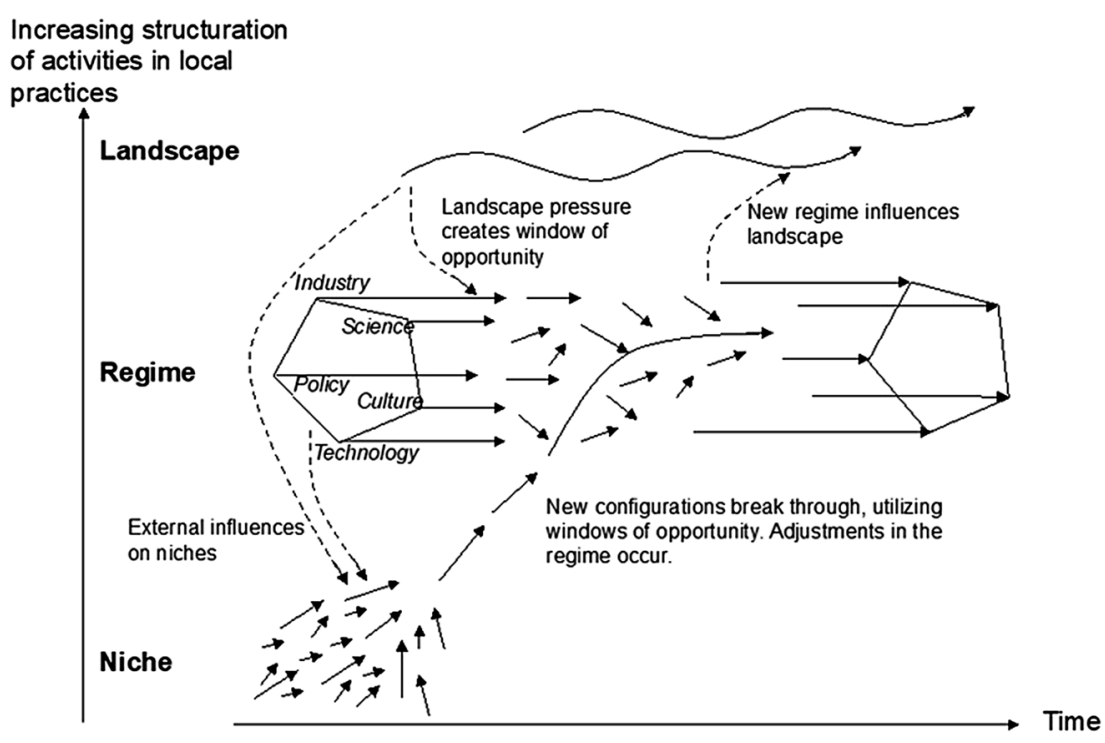

Abbildung 1 Multi-Level-Perspektive

Quelle: Grin/Rotmans/Schot (2010: 25) 


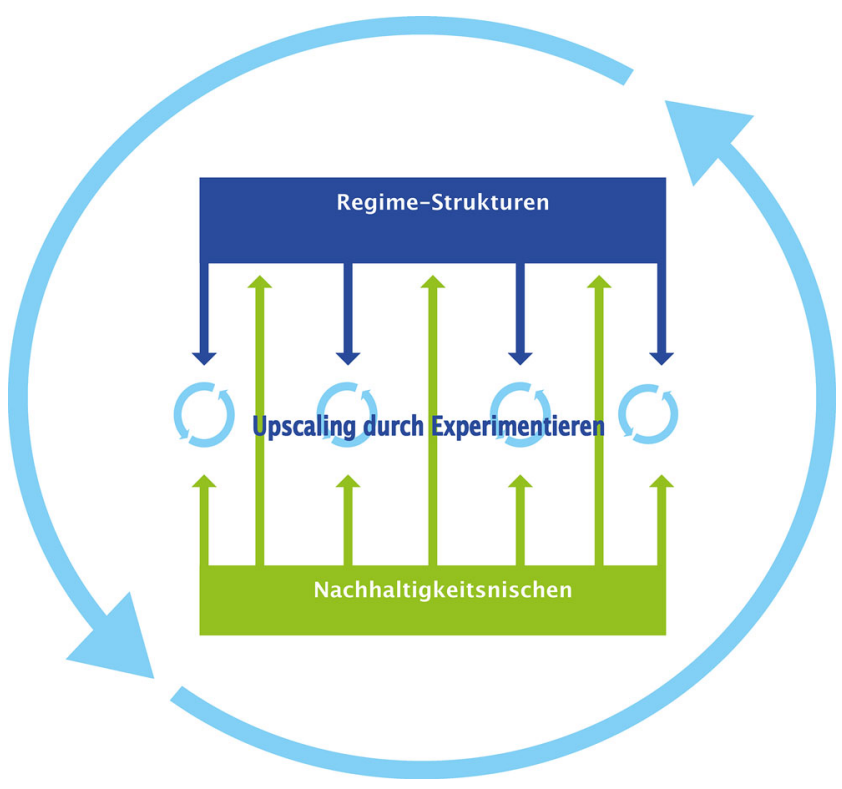

Abbildung 2 Experimente als reflexiver Prozess sich wandelnder Beziehung zwischen Regime und Nischen

Quelle: Eigene Darstellung, basierend auf Augenstein/Bachmann/ Hermelingmeier et al. (2020: 6)

das System insgesamt beeinflussen (Grin/Rotmans/Schot 2010: 18 ff.; Geels 2011).

Die Schaffung und das Schützen von Nischen als Keimzellen für die Entwicklung nachhaltiger Alternativen bildet einen zentralen Fokus in der Transition-Forschung (Kemp/ Schot/Hoogma 1998; Raven 2005; Schot/Geels 2008). In geschützten Nischen werden technologische und soziale Innovationen entwickelt, um damit zur Veränderung nichtnachhaltiger Strukturen und Praktiken im Regime beizutragen. Eine der Schlüsselfragen hierbei ist, wie das Upscaling, also der Durchbruch technologischer und sozialer Nischeninnovationen proaktiv gefördert und verbreitet werden kann (Ehnert/Kern/Borgström et al. 2018; von Wirth/Fuenfschilling/ Frantzeskaki et al. 2019; Lam/Martín-López/Wiek et al. 2020: Loorbach/Wittmayer/Avelino et al. 2020). Basierend auf den unterschiedlich ausgeprägten Strukturationsgraden in den Ebenen der Multi-Level-Perspektive und der Annahme kritik- und reflexionsfähiger Akteure ist tiefgreifender Wandel und das Upscaling von Alternativen grundsätzlich möglich. Allerdings bleibt es anspruchsvoll, insbesondere wenn anerkannt wird, dass Transformationsprozesse nicht linear, sondern koevolutionär verlaufen, da Routinen, Pfadabhängigkeiten und Machtverhältnisse in einem komplexen Wechselspiel zwischen ökonomischen, politischen und kulturellen Normen und Regelsystemen gewachsen und entsprechend verfestigt sind. Proaktive Versuche von Politik, Wirtschaft oder Zivilgesellschaft, bestimmte Nischeninnovationen strategisch zu fördern und zu skalieren, greifen deshalb oft zu kurz (Augenstein/Bachmann/Egermann et al. 2020).

Vor diesem Hintergrund erfordert ein Upscaling nachhaltiger Alternativen aus der Nische heraus weniger die Identifikation nachhaltiger Innovationen und deren lineare Skalierung, sondern eher einen reflexiven Lern- und Anpassungsprozess, der die dynamischen Wechselwirkungen zwischen nachhaltigkeitsorientierten Nischen und nichtnachhaltigen Regimestrukturen für Transformationsprozesse nutzbar macht (vgl. Abbildung 2). Experimente können dabei eine zentrale Rolle spielen, denn experimentelle Settings, beispielsweise in Reallaboren, schaffen einen Raum für die kritische Reflexion bestehender Strukturen und ermöglichen es, kreative Lösungsansätze zu entwickeln, diese immer wieder anzupassen und im Prozess daraus zu lernen. Eine solche experimentelle Herangehensweise fokussiert damit Such-, Lern- und Aushandlungsprozesse auf dem Weg hin zu einer nachhaltigeren Entwicklung, anstatt davon auszugehen, dass Nachhaltigkeit statisch definiert, umgesetzt und skaliert werden könne. Entsprechend werden in der Transition-Forschung Experimente definiert als ,inclusive, practice-based and challenge-led initiative designed to promote system innovation through social learning under conditions of uncertainty and ambiguity" (Sengers/Wieczorek/Raven 2019: 161). Darunter fallen dann vor allem urbane Living Labs (Nevens/Frantzeskaki/Gorissen et al. 2013; Bulkeley/ Marvin/Voytenko Palgan et al. 2019; Raven/Sengers/Spaeth et al. 2019) und Reallabore, die sich auf eine transdisziplinäre Wissensproduktion von Wissenschaft und Praxis konzentrieren und meist in Städten oder Quartieren durchgeführt werden (Schneidewind/Scheck 2013; Schäpke/Stelzer/ Caniglia et al. 2018; Wanner/Hilger/Westerkowski et al. 2018).

In diesem Beitrag werden Reallabore als reflexive Experimentier- und Lernräume betrachtet und institutionelle Logiken und Narrative als konzeptionelle Zugänge zu deren Analyse eingeführt. Dazu gibt das folgende Kapitel zunächst einen Überblick über den Reallaborbegriff und ein spezifisches Verständnis von Reallaboren als reflexive Experimentier- und Lernräume - vor dem Hintergrund des hier beschriebenen Transformationsverständnisses. In Kapitel 3 werden institutionelle Logiken und Narrative als analytische Konzepte eingeführt, mit deren Hilfe reflexive Lernprozesse in Reallaboren untersucht werden können. Dieser konzeptionelle Zugang wird dann in Kapitel 4 anhand einer Fallstudie aus Wuppertal illustriert. In Kapitel 5 werden anhand des Fallbeispiels methodische Schlüsselmerkmale und das spezifische Verständnis von Experimenten, ihrer Funktion und Auswirkungen im Kontext von Reallaboren diskutiert. Diese werden in der abschließenden Zusammenfassung in Bezug gesetzt zu experimentellen Ansätzen in der Planungswissenschaft und jüngeren Forderungen nach 


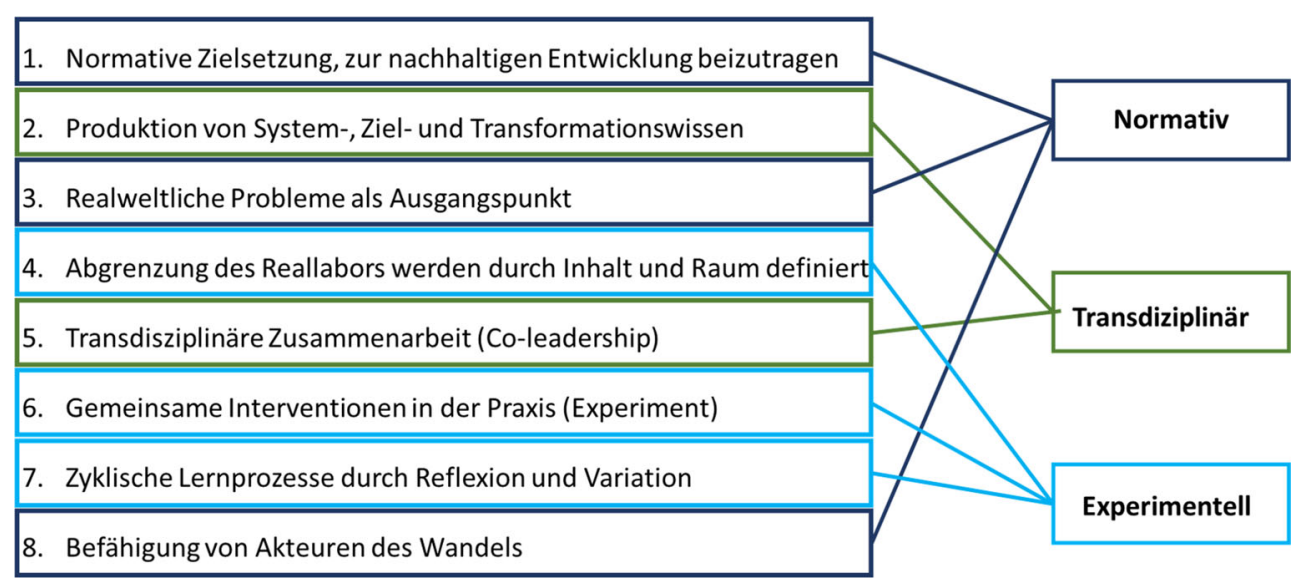

Abbildung 3 Charakteristika von Reallaboren

Quelle: Eigene Darstellung, basierend auf Wanner, Hilger, Westerkowski et al. (2018: 102) und

Schneidewind/Augenstein/Stelzer et al. (2018: 14)

dem vermehrten Einsatz experimenteller Ansätze auch in der Stadt- und Raumplanung. Dabei werden die unterschiedlichen Ausgangspunkte und Erwartungen bezüglich des Einsatzes von Experimenten in der Planung sowie in der Reallaborforschung beleuchtet (unter anderem in Bezug auf unterschiedliche Nachhaltigkeits- und Transformationsbegriffe) und potenzielle Möglichkeiten für wechselseitiges Lernen zwischen den beiden Forschungsbereichen diskutiert.

\section{Reallabore als reflexive Lernräume urbaner Transformationen}

Reallabore sind Räume oder Kontexte, in denen Forscher/-innen gemeinsam mit Akteuren aus der Praxis in einem transdisziplinären Forschungsprozess Wissen schaffen (WBGU 2016: 512). Unter anderem Schäpke, Stelzer, Bergmann et al. (2017) zeigen, dass Reallabore auf einigen Kernmerkmalen basieren, auch wenn das relativ junge Konzept und die Methode stetig weiterentwickelt werden. Dieser Beitrag folgt der Definition von Wanner, Hilger, Westerkowski et al. (2018), die Reallabore anhand von acht Merkmalen spezifizieren:

1) Normative framing: aiming to contribute to sustainable development;

2) Production of systems-, target-, and transformation knowledge;

3) Real-world problems as a starting point;

4) Boundaries: Laboratory demarcations, defined by content and space;

5) Transdisciplinary collaboration (co-leadership) with clear roles for practice and science;

6) Real-world intervention (experiment);
7) Cyclical learning processes through reflection and variation;

8) Empowerment of change agents and capacity building (Wanner/Hilger/Westerkowski et al. 2018: 107).

Wanner, Hilger, Westerkowski et al. (2018: 107) verstehen Reallabore damit als einen spezifischen Forschungsansatz, der die Grundprinzipien nachhaltigkeitsorientierter transdisziplinärer Forschung mit einem expliziten Augenmerk auf das Experiment als Methode der Wissensproduktion verbindet. Abbildung 3 fasst diese Grundprinzipien von Reallaboren in einem übergeordneten Dreiklang zusammen: Sie sind (1) transdisziplinär, sodass nicht nur theoretisches, wissenschaftliches Wissen generiert wird, sondern sowohl Systemwissen über den aktuellen Zustand und die Ausgangssituation, Zielwissen im Hinblick auf die wünschenswerte zukünftige Dimension als auch Transformationswissen über die möglichen Wege, wie man von der aktuellen Situation zu einer gewünschten nachhaltigeren gelangen kann. Diese transdisziplinäre Vorgehensweise beinhaltet außerdem sowohl eine besondere Herausforderung bei der Integration des Wissens verschiedener akademischer Disziplinen als auch des Fachwissens aus der Praxis. Gleichzeitig ist es wichtig, klare und gleichberechtigte Rollen für Forschende und Praxisakteure in der Zusammenarbeit festzulegen, sodass ein reines ,Beforschen“ der Praxis vermieden wird. Nur durch Kooperation auf Augenhöhe lässt sich ,sozial robustes Wissen“ (Nowotny 2003) gewinnen, das zur Lösung des praktischen Ausgangsproblems beiträgt und gleichzeitig relevantes wissenschaftliches Wissen erzeugt. Was das Reallabor als spezifischen Ansatz in der transdisziplinären Forschung außerdem charakterisiert, ist sein (2) experimenteller, räumlich und/oder inhaltlich abgegrenzter Laborcharakter. Interventionen und Experimente vor Ort sind der 
zentrale Modus der Wissensproduktion, indem mögliche Lösungen gemeinsam von Akteuren aus Wissenschaft und Praxis entwickelt, erprobt, reflektiert und variiert werden, sodass ein zyklischer Lernprozess stattfinden kann. Reallabore sind darüber hinaus explizit (3) normativ, da sie darauf abzielen, einen Beitrag zur nachhaltigen Entwicklung zu leisten und ein real-weltliches Problem als Ausgangspunkt für den transdisziplinären Forschungsprozess nehmen. AuBerdem wird angestrebt, transformative Wirkung über die räumlichen und zeitlichen Grenzen des Reallabors hinaus zu entfalten. Daher wird auch der Aufbau von Kapazitäten und die Befähigung von Akteuren vor Ort als Ziel oder Merkmal von Reallaboren definiert.

Während sich Wanner, Hilger, Westerkowski et al. (2018) auf den zyklischen Prozess der Forschung in Reallaboren konzentrieren, fügen wir eine strukturelle Perspektive auf Reallabore hinzu (Schneidewind/Augenstein/Stelzer et al. 2018). Diese Perspektive konzentriert sich deutlicher auf die Struktur, in die die zyklischen Lernprozesse eingebettet sind. Unter Struktur lässt sich hier einmal materielle Struktur verstehen, wie die physische Infrastruktur eines Reallabors, aber auch weitere sichtbare Strukturen, wie die Konstellation der beteiligten Akteure oder der Zugang zu finanziellen oder materiellen Ressourcen. Aufbauend auf Giddens (1984) sind hier aber auch die immateriellen oder virtuellen Strukturen berücksichtigt, die ein Reallabor charakterisieren, das heißt die Normen, Regeln und Interpretationsschemata, auf die beteiligte Akteure zurückgreifen, wenn sie Probleme identifizieren, relevante Fragen definieren und Lösungen entwickeln. Eine strukturelle Perspektive auf Reallabore geht also über die Idee eines Reallabors als eine rein physische Infrastruktur hinaus und nimmt die hinter dem Akteurhandeln liegenden Regeln und Logiken mit auf.

In Übereinstimmung mit Giddens' Konzept der Dualität von Struktur und Handeln, die auch der Multi-Level-Perspektive zugrunde liegt, ist diese Perspektive von zentraler Bedeutung: Unter der Annahme, dass Akteure grundsätzlich in der Lage sind, ihr Verhalten und die Art und Weise, wie sie sich auf bestimmte Regeln, Normen und Deutungsschemata stützen, zu reflektieren, verfügen sie auch über die Fähigkeit und das Potenzial, strukturelle Bedingungen zu hinterfragen und bewusst und absichtlich vom normalen, routinierten Verhalten abzuweichen und innovative Lösungen zu entwickeln (Schneidewind/Augenstein/Stelzer et al. 2018: 13). Aus struktureller Perspektive sind Reallabore damit Orte, in denen solche reflexiven Lernprozesse explizit angestrebt und ermöglicht werden. Ein solcher struktureller Zugang bietet die Möglichkeit, zu verstehen, wie genau sich die beteiligten Akteure zu bestehenden Strukturen positionieren, wie sie diese wahrnehmen, wie sie ihre alternativen Praktiken entwickeln und wo sie Raum für Verände- rungen und ihr eigenes Veränderungspotenzial sehen. Einer solchen interpretativen Perspektive folgend nehmen wir an, dass die Art und Weise, wie Akteure ihre Welt wahrnehmen, entscheidend dafür ist, wie sie handeln, mit anderen interagieren und damit die Welt gestalten. Dieser Dynamik und Dialektik zwischen Interpretation und Handlung folgend, lassen sich in Reallaboren kreative Lösungen und (Denk-)Ansätze entwickeln, die bestehende (nichtnachhaltige) Strukturen kritisch reflektieren, bewusst herausfordern und so Hebelpunkte für gesellschaftliche Transformation identifizieren.

\section{Analytische Perspektive: institutionelle Logiken und Narrative}

Um die Strukturierungsprozesse in Reallaboren und die der spezifischen, im nächsten Kapitel vorgestellten Fallstudie analysieren zu können, greifen wir auf das Konzept der institutionellen Logiken zurück sowie auf die Untersuchung von Narrativen der beteiligten Akteure, um zu verstehen, wie die bestehenden und alternativen Strukturen wahrgenommen und wie diese in Handlungen umgesetzt werden.

Institutionelle Logiken sind definiert als ,the socially constructed, historical patterns of material practices, assumptions, values, beliefs, and rules by which individuals produce and reproduce their material subsistence, organize time and space, and provide meaning to their social reality“ (Thornton/Ocasio 1999: 804). Sie sind gesellschaftlich anerkannte Grundprinzipien, die Akteuren helfen, Situationen angemessen zu interpretieren und erfolgreich zu handeln und drücken durch ihre Stärke den Grad der Strukturation, das heißt den Grad der Bindung des Handelns an dominante Strukturen in Nischen und Regime aus (Fuenfschilling/Truffer 2014: 774). Das Konzept versucht so eine Verbindung zwischen übergeordneten institutionellen Regeln, den Akteuren und deren Handlung herzustellen (Thornton/Ocasio 1999). Institutionelle Logiken erscheinen den jeweiligen Akteuren meist selbstverständlich, sodass sie oft fraglos oder unbewusst übernommen und mit ihren jeweiligen Legitimations-, Autoritäts- und Identitätsmustern reproduziert werden (Thornton/Ocasio/Lounsbury 2012: 73). Sowohl Akteure als auch Organisationen sehen sich oft mit multiplen und konkurrierenden Logiken konfrontiert, die ihr Handeln beeinflussen (Thornton/Ocasio 2008: 111). Die Vereinbarung dieser, teilweise widersprüchlichen, Logiken, fordert die Akteure einerseits heraus, eröffnet aber auch neue Interpretations- und Handlungsspielräume, die zu einer Veränderung von Institutionen führen können, worauf auch die Arbeiten der Institutional Work (Lawrence/Suddaby 2006) und des Institutional Entrepre- 
neurship (Greenwood/Suddaby 2006) hinweisen. Damit lassen sich aus einer Transformationsperspektive widersprüchliche Logiken und die Entstehung von Diskrepanzen in einem konkreten Umfeld als Potenzial verstehen, dominante und als gegeben angenommene Strukturen zu destabilisieren und Transformationspotenziale zu schaffen.

Ausgehend von der Dualität von Struktur und Handeln (Giddens 1984) bietet das Konzept der institutionellen Logiken daher eine analytische Perspektive darauf, wie das Handeln verschiedener Akteure in gegebenen Strukturen begründet ist, wie hier Struktur und Handlung miteinander in Beziehung stehen und wie sich Veränderungen auf Strukturebene nachzeichnen lassen (Thornton/Ocasio/Lounsbury 2012). So bietet das Konzept der institutionellen Logiken die Möglichkeit zu beleuchten, wie sich widersprüchliche Logiken um einen bestimmten Ort herum akkumulieren und den Prozess der Entwicklung eines lebenswerten urbanen Freiraums beeinflussen.

Um die institutionellen Logiken empirisch sichtbar zu machen, können Narrative analysiert werden, die die unterschiedlichen Wahrnehmungen der beteiligten Akteure beleuchten. Narrative können helfen zu verstehen, wie die Akteure institutionelle Logiken in ihr Handeln übersetzen, Legitimität für die bestehenden Logiken generieren und gemeinsame Identitäten konstruieren (Thornton/Ocasio/ Lounsbury 2012: 154f.). Sie bilden darüber hinaus ab, wie reflexive Lernprozesse in Reallaboren zur Reinterpretation von dominanten Strukturen führen und wie durch neu entstehende Narrative auch alternative Praktiken mit Bedeutung und Legitimität versehen werden. Narrative sind dabei definiert als mündliche oder schriftliche „Präsentation einer Abfolge von Ereignissen“ (,symbolic presentation of a sequence of events“, Scholes 1981: 205). Sie können als ein Medium angesehen werden, durch das die Akteure Komplexität reduzieren und mit Unsicherheit umgehen. Indem Menschen Geschichten erzählen, schaffen sie Kohärenz und Ordnung in ihren Wahrnehmungen und Erfahrungen. Dies geschieht, indem bestimmte Ereignisse oder Elemente einer Geschichte zu einer Abfolge von Ereignissen, einer chronologischen Reihenfolge (Anfang, Mitte und Ende einer Geschichte) angeordnet und durch ein kausales Muster oder eine logische Handlungsweise miteinander verbunden werden. Das Erzählen ist somit eine sehr grundlegende menschliche Tätigkeit, die zur Interpretation und Strukturierung der Realität eingesetzt wird (Prince 1982; Bruner 1990; Somers/Gibson 1993; Czarniawska 1998; Pentland 1999). Nicht rein literarische Narrative (,Wirklichkeitserzählungen“) charakterisiert, dass sie ein konstruktives und referenzielles Element enthalten: Sie interpretieren und konstruieren die Wirklichkeit, während sie gleichzeitig auf eine erlebte Realität und eine konkrete soziale Praxis verweisen (Klein/Martinez 2009: 1). Die Herausforderung besteht darin, diese beiden Aspekte miteinander in Beziehung zu setzen und zu untersuchen, wie die narrative Produktion von Wirklichkeit mit Handlungen in konkreten sozialen Kontexten interagiert (Hajer 1993). Ausgegehend von Sharp (2018) und Wittmayer, Backhaus, Avelino et al. (2015) sind Narrative hier ein guter Zugang: ,,[n]arratives are key to making sense of how frames are applied through storytelling to enact social change" (Sharp 2018: 519). Der Prozess des Erzählens selbst also kann als eine soziale Praxis verstanden werden, als ein kreativer Akt, der das Potenzial hat, diskursive Strukturen zu verändern (Gadinger/ Jarzebski/Yildiz 2014). In Bezug auf die Multi-Level-Perspektive konzentriert sich eine narrative Analyse auf die Art und Weise, wie Regimestrukturen und deren Logiken durch Sprache in dominanten Erzählungen reproduziert werden, während sie gleichzeitig die Dualität von Handlung und Struktur (Giddens 1984) betont, indem sie untersucht, wie Akteure auf dominante Logiken zurückgreifen und diese kreativ durch alternative Geschichten neu interpretieren.

\section{Illustration der Methode anhand der Fallstudie im Reallabor Wuppertal}

Die hier vorgestellte illustrative Fallstudie ist ein Experiment, das im Reallabor Wuppertal durchgeführt wurde. Das Reallabor Wuppertal ist eine Forschungsinfrastruktur, die seit 2015 durch Forschende und Akteure entwickelt wurde. Es erstreckt sich über mehrere Forschungsprojekte und konzentriert sich auf spezifische Fragen des urbanen Wohlstands und der nachhaltigen Stadtentwicklung in verschiedenen Stadtteilen und der Stadt insgesamt. ${ }^{1}$ Das hier betrachtete Experiment wurde gemeinsam mit Forschenden des Forschungsprojekts „UrbanUp“ ${ }^{\text {“2 }}$ und der zivilgesellschaftlichen Initiative „Utopiastadt“ durchgeführt. Utopiastadt befasst sich unter anderem mit Themen nachhaltiger Stadtentwicklung und konzentriert sich vor allem auf das Mirker Quartier in der Wuppertaler Nordstadt, in dem die Initiative angesiedelt ist. ${ }^{3}$ Eine Hauptaktivität von Utopiastadt ist die Restaurierung des alten Bahnhofsgebäudes (,Mirker Bahnhof") und dessen Entwicklung zu einem Katalysator für experimentelle, nachhaltige und integrative Stadt- bzw. Stadtteilentwicklung (vgl. Abbildung 4). In diesem Rahmen wurden in den letzten Jahren kulturelle Veranstaltungen und nachhaltigkeitsorientierte Aktionen, von Foodsharing und Urban Gardening über Fahrradverleih und Fahrradrepara-

\footnotetext{
1 http://www.urbanup.uni-wuppertal.de/ (22.03.2021).

2 http://www.urbanup.uni-wuppertal.de/ (22.03.2021).

3 https://clownfisch.eu/utopiastadt/ (22.03.2021).
} 


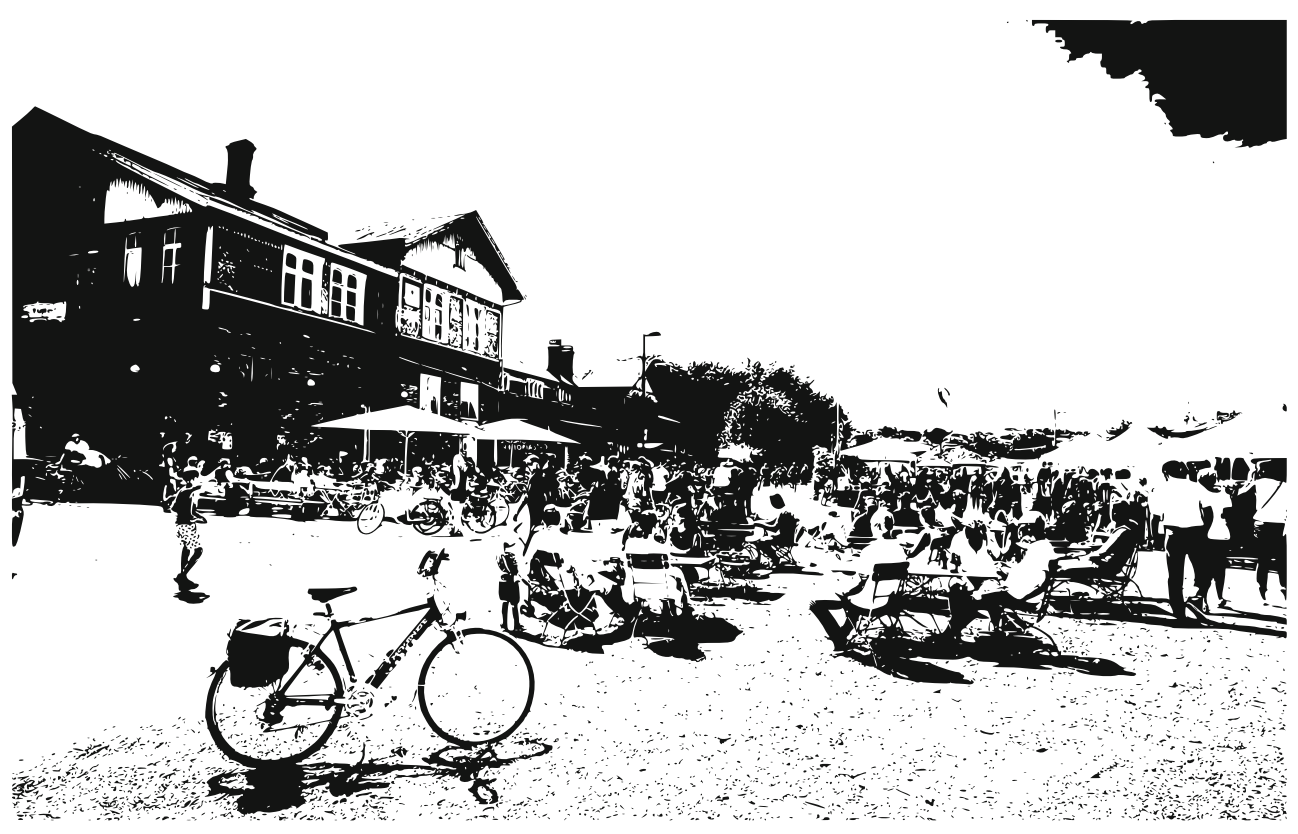

Abbildung 4 Utopiastadt, Mirker Bahnhof Foto: Wolf Sondermann

tur bis hin zu offenen Werkstätten, Co-Working und einem Hackerspace, etabliert. Im Jahr 2019 hat Utopiastadt eine Brachfläche neben dem alten Bahnhof erworben und ist dabei, diese als „Utopiastadt Campus“ im Sinne eines lebenswerten urbanen Freiraums zu entwickeln, diesen jedoch auch vor Gentrifizierungsprozessen zu bewahren und die Lebensqualität in der Stadt und dem Quartier zu erhöhen.

Um diese Form der Flächenentwicklung zu unterstützen, sollte im größeren Kontext des Reallabors Wuppertal ein Experiment durchgeführt werden. In einer Reihe von gemeinsamen Workshops im transdisziplinären Kernteam aus Forschenden und Mitgliedern von Utopiastadt wurden zunächst ein gemeinsames Problemverständnis entwickelt und gemeinsame Forschungsfragen erarbeitet. Diese Schritte waren unter anderem wichtig, um Vertrauen in der transdisziplinären Zusammenarbeit aufzubauen und die geplante Co-Leadership in der Durchführung des Experiments zu etablieren. Die gemeinsamen Forschungsfragen wurden so formuliert, dass sie sowohl den praktischen Bedürfnissen von Utopiastadt und den Interessen der beteiligten Forschenden Rechnung trugen: Wie kann urbaner Freiraum entwickelt werden, um dort gemeinwohlorientierten Ideen und Konzepten Raum zu bieten? Wie können Akteure wie Utopiastadt ihre gemeinwohlorientierten Ziele verwirklichen und gleichzeitig in ihrer Rolle als Grundstückseigentümerin mit wirtschaftlichen Zwängen und institutionellen Rahmenbedingungen umgehen? Wie können Akteurgruppen einbezogen werden, die bisher wenig Berührungspunkte mit der Initiative Utopiastadt hatten oder an der Entwicklung des
Utopiastadt-Campus beteiligt waren, und welche Spannungen oder Konflikte sowie positive Lernprozesse werden dadurch ausgelöst?

Um diese Fragen zu beantworten, wurde ein Experiment entwickelt und gemeinsam durchgeführt: Auf dem neu gekauften Campusgelände wurden zusätzlich zu den bereits bestehenden Aktionen, die in alten Schiffscontainern angeboten wurden (z. B. Fahrradverleih und -reparatur, gastronomische Angebote) zwei weitere Schiffscontainer zur Verfügung gestellt und für kurzfristige experimentelle Nutzungen vermietet, um verschiedene Konzepte zur Belebung des Raumes auszuprobieren. Über diverse Kanäle wurde ein offener Aufruf zur Teilnahme gestartet, der allen, die eine geschäftliche, soziale, kulturelle, künstlerische oder andere Idee für den Utopiastadt-Campus hatten, die Möglichkeit bot, einen der Container für einen Zeitraum von bis zu acht Wochen für einen Gegenwert von einem Euro zu nutzen. Das Experiment lief von Juni bis Oktober 2019 und die Container wurden von einer Vielzahl von Akteuren, lokalen Unternehmen, Künstlerinnen/Künstlern, Existenzgründerinnen/-gründern, Sozialunternehmerinnen/-unternehmern und einem Kommunalpolitiker genutzt.

Das übergeordnete Ziel des Experiments bestand darin, zunächst die unterschiedlichen Bedeutungen und Funktionen, die dem Campusgelände rund um den Mirker Bahnhof und der dort ansässigen Initiative Utopiastadt zugeschrieben werden, zu erfassen und darauf aufbauend einen Reflexionsund Lernprozess anzustoßen für eine gemeinwohlorientierte Entwicklung des Utopiastadt-Campus. Dabei wurde ein wesentlicher Fokus auf die Rollen gelegt, die aus der Sicht 
der am Experiment Teilnehmenden, Besucher/-innen und Mitgliedern von Utopiastadt unterschiedliche urbane Akteure in dem Entwicklungsprozess einnehmen könnten oder sollten. Grundlage der Analyse waren 39 halbstrukturierte Interviews mit Teilnehmenden des Container-Experiments und Besucherinnen/Besuchern der Fläche. Die Interviews geben Einblicke in die Bedeutungen und Funktionen, die Utopiastadt als Ort (Mirker Bahnhof und Campus-Fläche) zugeschrieben werden, in die Motivation und Erfahrungen der Teilnehmenden am Experiment sowie in allgemeine Vorstellungen über nachhaltige Stadtentwicklung. Die Interviews dienten der Konstruktion von Narrativen über die Entwicklung lebenswerter urbaner Freiräume und wurden im Hinblick auf die unterschiedlichen institutionellen Logiken analysiert, auf die die Interviewten in ihren persönlichen Berichten zurückgreifen. Die Beobachtungen durch die beteiligten Forschenden vor Ort lieferten zusätzliche Einblicke in die Motivation und Anliegen der Teilnehmenden des Experiments und der Besucher/-innen und dienten der Triangulation der Ergebnisse. Die Analyse der Daten basiert auf der Inhaltsanalyse nach Mayring (2015), in der die Daten kodiert und kategorisiert wurden. Aus dem Material wurden Narrative konstruiert, wobei der Schwerpunkt auf dem Emplotment, das heißt der Gesamthandlung einer Erzählung als solcher, und auf der Art und Weise lag, wie eine bestimmte Problemstellung und die Hauptfiguren der Geschichten miteinander verbunden sind. $\mathrm{Zu}$ beleuchten, wie genau Problemsituationen im Hinblick auf Herausforderungen und Chancen konstruiert wurden und welche Rollen und Verantwortlichkeiten verschiedenen städtischen Akteuren bei der Lösung von Problemen zugewiesen wurden, kann dazu beitragen zu verstehen, welche Bedeutung bestimmten Orten beigemessen werden, welche Logiken in diesem Ort verknüpft sind und welche Wege der Transformation als möglich gesehen werden.

Der Zweck des Experiments bestand also nicht darin, konkrete Lösungen für die finanziell tragbare und trotzdem gemeinwohlorientierte Entwicklung der neuen Fläche hin zu einem urbanen Freiraum zu finden, die dann konkret umgesetzt werden kann. Es ging vielmehr im Sinne des oben beschriebenen Upscaling darum zu untersuchen, wie mithilfe des durchgeführten Experiments Spannungen zwischen institutionellen Logiken bei zivilgesellschaftlichen Initiativen wie Utopiastadt, die sich als zunehmend relevante Akteurgruppe in nachhaltiger Stadtentwicklung erweisen, identifizieren und reflektieren lassen, welche unterschiedlichen Funktionen lebenswerten urbanen Freiraums von dessen (potenziellen) Nutzerinnen und Nutzern wahrgenommen werden und wie Prozesse nachhaltiger Stadtentwicklung aus der Sicht der beteiligten Akteure zukünftig gestaltet werden könnten oder sollten.

Die Ergebnisse des Experiments wurden im transdis- ziplinären Team und gemeinsam mit den Teilnehmenden des Container-Experiments abschließend reflektiert. Ursprünglich war geplant, auf den gemachten Erfahrungen aufzubauen und das Experiment entsprechend angepasst fortzusetzen, allerdings hat sich diese Weiterentwicklung aufgrund der Corona-Pandemie und der damit verbundenen Einschränkungen verzögert.

\section{Diskussion der Ergebnisse: Rolle von zivilgesellschaftlich entwickelten Orten in der urbanen Transformation}

Im Folgenden werden einige der wichtigsten Ergebnisse der narrativen Inhaltsanalyse vorgestellt. Um die gemeinsame Forschungsfrage nach der gemeinwohlorientierten Entwicklung lebenswerten urbanen Freiraums zu beantworten, wurde unter anderem analysiert, was die spezifische Qualität des Ortes Utopiastadt (Mirker Bahnhof und CampusFläche) aus der Perspektive seiner Nutzer/-innen ausmacht. Aus der Codierung und Kategorisierung des Materials ließen sich sieben übergreifende Kategorien von Funktionen ableiten, die dieser Ort erfüllt:

1. ein Kreativ- und Freiraum, eine „Keimzelle, wo Dinge einfach ausprobiert werden",

2. ein offener Ort der Begegnung, der zur Integration beiträgt: „Die Atmosphäre ist einfach toll. Leute begegnen sich hier. Ich hab' hier schon viele Freundschaften geschlossen",

3. ein Ort, an dem man ausgehen und gastronomischen Service genießen kann, als ,einziger Ort sag ich mal hier in der Umgebung, wo man öffentlich mit Leuten treffen kann, was trinken kann, gemeinsam chillen kann und ab und zu mal gibt's hier auch Musik und nen Konzert",

4. ein zentraler Vernetzungsort für Künster/-innen, die Kulturszene und Nachhaltigkeitsinitiativen, also ein Ort ,an dem zentriert sich das hier so irgendwie (...) und das ist halt sehr gut, weil man weiß, man kommt hier hin und da ist alles irgendwie, muss nicht suchen",

5. ein Ort mit hoher Aufenthaltsqualität ohne die Notwendigkeit konsumieren zu müssen, ,,wo du auch mal hinkommen kannst, dir auch mal eine Liege nehmen kannst, ohne irgendwie was zu kaufen",

6. ein Ort des Rückzugs und der Erholung für die Bewohner/-innen, ,so im positiven Sinne eine Oase, um sich auch mal aufzutanken, genau mit der Energie hier",

7. ein Erhohlungsort, der auch Touristen anzieht und zur Verbesserung des allgemeinen Images der Stadt beiträgt: 
„immer wenn man hier Leute hinbringt, sagen die, oooh Wuppertal ist ja eigentlich ganz cool“".

Dieser kurze Überblick zeigt, dass das, was den von Utopiastadt belebten Raum zu einem attraktiven und wertvollen Ort für seine Nutzer/-innen macht, seine spezifische Rolle als (halb)öffentlicher Raum ist, der sich beispielsweise von den eher konsumorientierten Innenstädten, aber auch von rein natur- oder unterhaltungsorientierten Erholungsgebieten wie Parks oder Spielplätzen unterscheidet. Utopiastadt wird als ein Ort verstanden, der eine öffentliche oder soziale Funktion übernimmt, es ist ,ein möglichkeitsschaffender Raum, der irgendwie probiert auch eine Lücke zu füllen“, „aber nicht Stadt. Wenn ich Stadt will, dann gehe ich hier runter". Er wird als ein Ort der Begegnung wahrgenommen, an dem sich Menschen in kulturellen oder nachhaltigkeitsorientierten Tätigkeiten entfalten können. Auch lassen sich die unterschiedlichen institutionellen Logiken bezüglich der Abgrenzung zwischen öffentlichem und privatem Raum erkennen, die sich insbesondere zwischen einer ökonomischkommerziell und gemeinwohlorientierten Logik überschneiden: Während in einigen Interviews betont wurde, dass die Qualität dieses Ortes genau in seinem schönen Ambiente mit gastronomischem Angebot liegt, besuchen viele andere den Ort hauptsächlich, da dieser als frei von Konsumzwängen wahrgenommen wird: ,als das Wort Geld gefallen ist, da war eine Dame, die hat auf dem Absatz umgedreht und ist gegangen“. Hier zeichnet sich ein Spannungsfeld ab, was die zentrale Herausforderung angeht, eine Balance zu finden zwischen kommerziell und konsumfrei, um die Bedürfnisse aller Besucher/-innen, Bewohner/-innen und auch der Initiative selbst zu erfüllen. Denn auch die Initiative Utopiastadt befindet sich als Organisation in einer Spannungssituation: Einerseits ist der eigene Anspruch, ein ,,andauernder Gesellschaftskongress mit Ambition und Wirkung “4 zu sein, andererseits besteht die Notwendigkeit, eine stabile finanzielle Basis für ihre Aktivität sicherzustellen.

Das Container-Experiment war ein Versuch herauszufinden, wo dieses Gleichgewicht zwischen kommerzieller und gemeinwohlorientierter Logik zu finden ist und wie sich gerade aus dem Widerspruch neue Handlungs- und Interpretationsräume eröffnen lassen. Der offene Aufruf an alle Interessierten, die beiden zur Verfügung gestellten Container zu nutzen, sollte zeigen, wer überhaupt bereit ist, mit seinen Ideen und Konzepten zur Entwicklung der CampusFläche beizutragen, was jeweils individuelle Beweggründe sind und wie diese mit der Gesamtidee eines gemeinwohlorientierten und lebenswerten urbanen Freiraums zusammen-

${ }^{4}$ https://clownfisch.eu/utopiastadt/ (08.03.2021). hängen. Die Teilnehmenden hatten vielfältige unternehmerische, künstlerische, soziale und politische Ziele, die sie mit ihrem Container-Experiment verfolgen wollten. Vor allem teilnehmende lokale Unternehmen verstanden den Ort als alternativen wirtschaftlichen Standort und als eine Art alternativen Marktplatz, auf dem sie ihre Produkte außerhalb ihres normalen Geschäfts verkaufen konnten, sodass sie ,mal rauskommen aus dem festen Laden; Spektrum erweitern, sowohl Kunden- als auch Wirtschaftlich“.

Was alle Teilnehmenden einte war die Einsicht, dass die Zeit im Container ihnen eine Möglichkeit zum Experimentieren außerhalb ihrer gewohnten Umstände bot. Der experimentelle Charakter erwies sich vor allem deshalb als attraktiv, weil das Projekt mit den minimalen Kosten und der zeitlichen Limitierung mit geringen Risiken verbunden war, denn ,in so einer harten Business-Umgebung (...) also wenn ich das jetzt irgendwo in der Innenstadt machen wollte und müsste erst zu einer Bank gehen, mir das Geld leihen und irgendwie (...) da hätte ich keine Lust zu“. Insbesondere in der Rückschau fanden die meisten Nutzer/ -innen den Punkt ,stark', dass die Teilnahme an diesem Experiment für sie nützlich war, um über ihre jeweiligen Ziele neu nachzudenken sowie die Art und Weise, wie sie normalerweise wirtschaften. So stellten die lokalen Unternehmer/ -innen heraus, dass die Zeit im Container eine wertvolle Erfahrung war, auch wenn das Experiment für sie selten ein wirtschaftlicher Erfolg war: „Das hat uns ja inspiriert, hier zu experimentieren. Also wir hätten das in unserem (...) Umfeld nicht gemacht, weil das ist da natürlich, da hätten wir viel wirtschaftlicher gedacht“.

Was zusammengefasst beobachtet werden konnte, war also eine Überschneidung unterschiedlicher Logiken, sowohl in der Motivation der Teilnehmenden aber auch in der Rückschau auf die Erfahrungen, die durchaus Spannungen, aber keine kritischen Konflikte zu erzeugen schienen: „Natürlich konnte man da jetzt nicht so gut arbeiten wie ich jetzt hier arbeiten kann. Und natürlich war es auch eine Doppelbelastung, weil ich ja trotzdem hier (arbeiten) musste. Aber grundsätzlich fand ich das eine coole Zeit. Und umsatzmäßig, ja, das kann man nicht bemessen“. Zur Einordnung dieser Ergebnisse ist es wichtig festzuhalten, dass die meisten Teilnehmenden aufgrund des offenen Aufrufs bereits mit Utopiastadt vertraut waren und sich allgemein für Nachhaltigkeit und Stadtentwicklung interessierten. Aus diesem Grund wurde zur Weiterentwicklung und Verstetigung des Experiments für 2020 geplant, die mögliche Containernutzung fortzusetzen und durch die aktive Einladung eines vielfältigeren Kreises zur Teilnahme, unter anderem auch mit traditionelleren Unternehmen, weiterzuentwickeln, was allerdings aufgrund der aktuellen Pandemiesituation verschoben werden musste.

Neben einer besseren Vorstellung von der Bedeutung und 
Funktion der Utopiastadt-Campus-Fäche und der Motivation derjenigen, die an einem Beitrag zu deren Entwicklung interessiert sind, wurden außerdem allgemeinere Vorstellungen zu wünschenswerten Prozessen nachhaltiger Stadtentwicklung untersucht. Aus dem Datenmaterial wurden Narrative konstruiert, die die Vorstellungen der Teilnehmenden am Experiment sowie von Utopiastadt-Besucherinnen/ Besuchern und Utopiastadt-Engagierten abbilden bezüglich der wahrgenommenen und wünschenswerten Rolle unterschiedlicher Akteurgruppen für eine nachhaltige Stadtentwicklung (z. B. lokale Politik, Stadtverwaltung, lokale Wirtschaft, zivilgesellschaftliche Initiativen und Bürger/-innen). Durch den Fokus auf die Utopiastadt-Campus-Flächen, die sich im Besitz einer zivilgesellschaftlichen Initiative befinden, ließ sich aus den Interviews ein dominierendes Narrativ zu Utopiastadt und der Campus-Fläche identifizieren, das die Herausforderungen der Entwicklung eines lebenswerten urbanen Freiraums (aus Sicht der Befragten) beschreibt. In diesem Narrativ wird Stadtentwicklung als eine komplexe Problemsituation wahrgenommen und beschrieben, in der „die (lokale) Politik“ eher negativ und als wenig proaktiv betrachtet wird. Aufgrund einer sehr schlechten finanziellen Situation sei die Stadt auf bürgerschaftliches Engagement und Beiträge der Bürger/-innen zur Stadtentwicklung angewiesen, sodass , viel auf Initiativen und Ehrenamt abgeschoben (wird), wenn kein Geld oder Interesse da ist, Dinge zu unterstützen“. Gleichzeitig seien viele Bürger/-innen nicht wirklich motiviert, sich zu engagieren, und diejenigen, die sich engagierten, seien frustriert, weil sie sich von der Kommunalverwaltung nicht unterstützt und nicht gehört fühlten: „die Gestaltungsmacht ist eben auch ungleich verteilt, da habe ich das Gefühl, ich habe keine Macht und deswegen ja, habe ich da auch nicht so die Motivation, überhaupt etwas zu machen“. Das Ideal oder die Vision für die Zukunft von Stadtentwicklung im Allgemeinen, das in dem übergeordneten Narrativ zum Ausdruck kommt, ist das einer Stadt, die nach einer anderen Logik funktioniert: eine, die von Bürgerinnen und Bürgern aktiv mitgestaltet wird und in der urbaner Raum nach den Bedürfnissen der Bewohner/ -innen entwickelt wird, unterstützt durch lokale Politiker/innen sowie die institutionellen Strukturen und Prozesse der Stadtverwaltung, sodass ,die Leute eine Idee finden und sagen, wir brauchen Hilfe von der Stadt, wir machen das jetzt zusammen“. In diesem Narrativ wird die Utopiastadt-Campus-Fläche als ein Beispiel für einen spezifischen Ort gesehen, der die Möglichkeit bietet, diese Vision umzusetzen, da „es hier tatsächlich so ist, dass Menschen etwas machen können, auch Macht haben in dem Sinne“. Es wird betont, dass Bürger/-innen genau solche Möglichkeiten brauchen, um sich zu engagieren, Selbstwirksamkeit zu erfahren und ein Verständnis für die politische Macht zu entwickeln, die sie entfalten können. Auf der Grundlage dieser Erfahrun- gen können Bürger/-innen ihre Bedürfnisse und ihre Bereitschaft, sich einzubringen, klarer formulieren und in einen Dialog mit der lokalen Politik und Verwaltung treten.

Die im Rahmen dieses Experiments gesammelten Narrative bieten so eine neue Perspektive auf die Rolle zivilgesellschaftlicher Initiativen in urbanen Transformationsprozessen. Sie geben Einblicke in die Bedeutung, die einem lebenswerten urbanen Freiraum von den beteiligten Akteuren beigemessen wird und generieren System-, Ziel- und Transformationswissen für die normativ gewünschte weitere Entwicklung des Ortes. Auch wenn Einsichten nur für diesen speziellen Fall relevant zu sein scheinen, lässt sich erkennen, dass bei Versuchen, die Komplexität des örtlichen Kontexts zu erfassen, der Einfluss der Bedeutungen des Orts berücksichtigt werden muss, da diese die lokale Wahrnehmung und Steuerung von Entwicklungsprozessen durchaus prägen (Hodson/Geels/McMeekin 2017; Hodson/ Marvin 2017). Stadtsoziologische Studien haben gezeigt, dass „differences between places in terms of discourses, cultural frames and identity result to be critical factors for transition governance“ (Wolfram/Frantzeskaki 2016: 8). So lassen sich durchaus aus Experimenten in Reallaboren, die notwendigerweise ortsspezifisch sind, allgemeine Erkenntnisse darüber ableiten, wie lokalspezifische Diskurse und Narrative im Verhältnis zu gesamtgesellschaftlichen Nachhaltigkeitsherausforderungen stehen. Im Reallabor Wuppertal und dem hier betrachteten Experiment kommt dieses Verhältnis in den Bezügen zwischen individuellen Narrativen der beteiligten Akteure und den von ihnen wahrgenommenen institutionellen Logiken und entsprechenden Konflikten (z. B. kommerziell vs. gemeinwohlorientiert) zum Ausdruck. Zivilgesellschaftliche Initiativen, die sich wie in diesem Fall auf unterschiedliche Logiken stützen oder beispielsweise sowohl markt- als auch gemeinwohlorientierte Logiken versuchen, in einen neuen Ausgleich zu bringen, können experimentell innovative Ansätze entwickeln, wie ein Gleichgewicht zwischen ihnen hergestellt werden kann: „da passieren auf einmal andere Sachen (...) die Ware, die da nun mit ins Spiel kommt, die kannst du gar nicht mehr so konkretisieren“. Hier entsteht dann auch ein UpscalingPotenzial, das sich nicht in der Skalierung einzelner Bestpractice-Beispiele erschöpft, sondern auf die Ermöglichung fortlaufender Reflexions- und Lernprozesse und das Entdecken von Handlungsspielräumen setzt. 


\section{Zusammenfassung: Reallabore als Zugang zu kontextspezifischem Wissen und Ort reflexiver Lernprozesse}

Anhand der Fallstudie aus dem Reallabor Wuppertal wurde aufgezeigt, wie Experimente helfen können, einen $\mathrm{Zu}$ gang zu kontextspezifischem Wissen zu schaffen. Anhand der Experimente können so die unterschiedlichen bestehenden Narrative, sowie deren dahinterliegenden Logiken, und die Bedeutungen eines Orts der urbanen Transformation für seine weitere Entwicklung verstanden und nutzbar gemacht werden. Ein wesentliches Ergebnis bestand darin, die unterschiedlichen Visionen und Ziele der beteiligten Akteure zu explizieren. Das betrachtete Experiment im Reallabor schuf einen reflexiven Lernraum, in dem die Spannungen, die sich aus unterschiedlichen Ansprüchen und Logiken ergeben, reflektiert wurden und produktiv zur Gestaltung des weiteren Transformationsprozesses genutzt werden können. In diesem Fallbeispiel wurde insbesondere die Rolle einer zivilgesellschaftlichen Initiative (Utopiastadt) in urbanen Transformationprozessen beleuchtet, die gemeinsam mit Wissenschaftlerinnen/Wissenschaftlern, lokalen Unternehmerinnen/Unternehmern, Künstlerinnen/Künstlern und Bürgerinnen/Bürgern einen experimentellen Entwicklungsprozess für lebenswerte urbane Freiräume initiierte und umsetzte.

Die explizite Einbeziehung zivilgesellschaftlicher Initiativen als relevante Träger lokalen Wissens ist in der urbanen Reallaborforschung zentral und stützt sich auf die Erkenntnis, dass diese Akteurgruppe zunehmend als relevanter Treiber urbaner Nachhaltigkeitstransformationen in den Vordergrund tritt (McPhearson/Wijsman 2017: 66). Dieses Potenzial kann insbesondere in Reallaboren berücksichtigt und gefördert werden, da hier zivilgesellschafltiche Initiativen gleichberechtigt an transdisziplinären Forschungsprozessen teilnehmen und ihr spezifisches Wissen einbringen. Im Hinblick auf die Förderung urbaner Nachhaltigkeitstransformationen fungieren Reallabore damit insbesondere als strukturierte Experimentier- und Reflexionsräume, um dominante institutionelle Logiken und (nichtnachhaltige) Strukturen sichtbar zu machen, zu hinterfragen und zur Diskussion zu stellen. Insbesondere in der transdisziplinären Zusammenarbeit und über die Integration verschiedener wissenschaftlicher und Praxis-Perspektiven können so alternative Wege für einen proaktiven und kreativen Umgang mit den Herausforderungen urbaner Transformation entwickelt und erprobt werden.

Jedoch zeigen sich in der Reallaborforschung auch Grenzen: Das Beispiel der zivilgesellschaftlichen Initiativen, die Stadt- und Quartiersentwicklung betreiben, wirft beispielsweise Fragen der demokratischen Legitimation und der Par- tizipation an diesen Prozessen auf, die kritisch diskutiert und hinterfragt werden müssen. Im Fallbeispiel von Utopiastadt findet die experimentelle Prozessgestaltung für die Entwicklung von urbanen Freiräumen auf dem Gelände des Utopiastadt-Campus selbst statt und damit wird nicht öffentlicher, sondern privater Raum entwickelt - jedoch mit einem gemeinwohlorientierten, gesellschaftlichen Anspruch. In anderen Fällen ,klassischer' Stadtplanung und der Entwicklung öffentlichen Raums nehmen Stadtplaner/-innen und die lokale Verwaltung als politisch legitimierte Akteure eine zentrale Rolle ein. Diese können dann zwar einen partizipativen Prozess initiieren, letztendlich obliegt ihnen aber die legitimierte Entscheidungsgewalt (Kanning 2018: 23). Eine Kombination von partizipativer Planung und experimentellen Ansätzen aus der Reallaborforschung könnte ein interessantes Potenzial bieten, die Möglichkeiten und Grenzen von bottom-up initiierten Stadtentwicklungsprozessen und der Rolle zivilgesellschaftlicher Initiativen zu reflektieren und neu auszuloten.

Als Basis dafür müssen jedoch zentrale Konzepte und ihr jeweils unterschiedliches Verständnis explizit gemacht werden. Das betrifft beispielsweise den Nachhaltigkeitsbegriff. In der Reallaborforschung wird Nachhaltigkeit als übergeordnetes, normatives Ziel verfolgt, welches allerdings vielschichtig, ambivalent und mit Unsicherheiten behaftet bleibt, sodass das Upscaling nachhaltiger Alternativen und die Gestaltung von Transformationsprozessen vor allem ein gemeinsamer Such- und Lernprozess ist. In der Planung herrscht laut Kanning (2018: 24) dagegen ein Nachhaltigkeitsverständnis vor, welches vor allem ökologische Kriterien in den Blick nimmt und weniger die damit verbundenen gesellschaftlichen Veränderungsprozesse und -anforderungen. An ein solch unterschiedliches Verständnis schließt sich auch ein unterschiedliches Verständnis für den Sinn und Nutzen von Experimenten an. In der Reallaborforschung werden Experimente vor dem Hintergrund komplexer und koevolutionärer Transformationsprozesse als nützlich erachtet, um die inhärenten Ambivalenzen und Unsicherheiten in Bezug auf eine nachhaltige Entwicklung zu reflektieren. Experimente sind in diesem Zusammenhang ein Ansatz, um verschiedene Arten von Wissen (System-, Transformations- und Zielwissen) und das Wissen unterschiedlicher Akteure (theoretisches Wissen der Wissenschaft und lokales Expertenwissen der Praxis) mit einzubeziehen, um sozial robustes Wissen zu produzieren und Transformationsprozesse aktiv zu gestalten. Hier lässt sich an bestehende experimentelle Ansätze in der Planung bzw. Planungswissenschaft anknüpfen, die ebenfalls an Prinzipien der Partizipation und des Co-Design ausgerichtet sind (Kanning 2018: 23). Ein wesentlicher Unterschied besteht jedoch darin, dass in der Reallaborforschung Experimente sowohl zur gemeinsamen Generierung von Wissen 
,auf Augenhöhe“ als auch zur praktischen Erprobung und Umsetzung dienen sollen. In der Planung sind die beteiligten Verwaltungsakteure jedoch in der Regel auch diejenigen, die formale Entscheidungshoheit haben und damit Interessens- und Machtkonflikten ausgesetzt sind (Kanning 2018: 24). In der Planungswissenschaft gibt es außerdem Forderungen nach dem vermehrten Einsatz von weniger umsetzungs- als eher wissenschaftlich orientierten Feldexperimenten (Honey-Rosés/Stevens 2019). Diese zielen auf Experimente in kontrollierten Umgebungen ab, in denen möglichst Variablen kontrolliert und Kontrollgruppen eingesetzt werden können, um so kausale Zusammenhänge aufzudecken und greifbare Auswirkungen zu messen.

Es bestehen also wesentliche Unterschiede zwischen verschiedenen Formen von Experimenten, die jedoch an der Schnittstelle von Reallaborforschung und Planung (insbesondere in konkreten urbanen Kontexten) gewinnbringend kombiniert eingesetzt werden könnten, wenn die jeweiligen Annahmen und Zielsetzungen entsprechend explizit gemacht werden. Experimente in Reallaboren stellen aus dieser Perspektive eine sinnvolle Ergänzung zu Ansätzen in der Planung dar, da hier der Fokus auf reflexiven Lernprozessen als Grundlage für komplexe Veränderungsprozesse liegt. Insbesondere die strukturierte Einbindung zivilgesellschaftlicher Initiativen kann, wie hier gezeigt, dazu beitragen kreative Perspektiven aufzudecken, die quer zu bestehenden politischen, administrativen und ökonomischen Logiken verlaufen. Damit ist ein Ziel von Reallaboren nicht unbedingt Blaupausen oder Strategien zu entwickeln, die in anderen Kontexten skalier- und anwendbar sind, sondern Raum für reflexives Lernen zu schaffen und damit ,handles for reflective practice that (...) can instigate accumulation of changes and increase the pace of changes" (McPhearson/Wijsman 2017: 68) bereitzustellen. Insbesondere zivilgesellschaftliche Initiativen können zu Treibern der urbanen Transformation werden (für einen Überblick vgl. (McPhearson/Wijsman 2017: 66), in vielen Fällen unterstützt und eingebettet in Experimente und zyklische Lernprozesse in Reallaboren.

\section{Literatur}

Augenstein, K.; Bachmann, B.; Egermann, M.; Hermelingmeier, V.; Hilger, A.; Jaeger-Erben, M.; Kessler, A.; Lam, D.P.M.; Palzkill, A.; Suski, P.; von Wirth, T. (2020): From niche to mainstream: the dilemmas of scaling up sustainable alternatives. In: GAIA - Ecological Perspectives for Science and Society 29, 3, 143-147. https://doi. org/10.14512/gaia.29.3.3

Augenstein, K.; Bachmann, B.; Hermelingmeier, V.; Kessler, A.; Palzkill, A.; Suski, P. (2020): The Dilemma of Upscaling. Wuppertal. = UrbanUp Working Paper II.
Bruner, J.S. (1990): Acts of Meaning. Cambridge.

Bulkeley, H.; Castán Broto, V.; Hodson, M.; Marvin, S. (Hrsg.) (2011): Cities and low carbon transitions. New York.

Bulkeley, H.; Marvin, S.; Voytenko Palgan, Y.; McCormick, K.; Breitfuss-Loidl, M.; Mai, L.; von Wirth, T.; Frantzeskaki, N. (2019): Urban living laboratories: Conducting the experimental city? In: European Urban and Regional Studies 26, 4, 317-335. https://doi.org/10.1177/ 0969776418787222

Coenen, L.; Benneworth, P.; Truffer, B. (2012): Toward a spatial perspective on sustainability transitions. In: Research Policy 41, 6, 968-979. https://doi.org/10.1016/j. respol.2012.02.014

Czarniawska, B. (1998): A narrative approach to organization studies. Thousand Oaks.

Ehnert, F.; Kern, F.; Borgström, S.; Gorissen, L.; Maschmeyer, S.; Egermann, M. (2018): Urban sustainability transitions in a context of multi-level governance: A comparison of four European states. In: Environmental Innovation and Societal Transitions 26, 101-116. https://doi. org/10.1016/j.eist.2017.05.002

Fuenfschilling, L.; Truffer, B. (2014): The structuration of socio-technical regimes. Conceptual foundations from institutional theory. In: Research Policy 43, 4, 772-791. https://doi.org/10.1016/j.respol.2013.10.010

Gadinger, F.; Jarzebski, S.; Yildiz, T. (2014): Politische Narrative. Konturen einer politikwissenschaftlichen Erzähltheorie. In: Gadinger, F.; Jarzebski, S.; Yildiz, T. (Hrsg.): Politische Narrative: Konzepte - Analysen - Forschungspraxis. Wiesbaden, 3-38. https://doi.org/10.1007/978-3658-02581-6_1

Geels, F.W. (2011): The multi-level perspective on sustainability transitions: Responses to seven criticisms. In: Environmental Innovation and Societal Transitions 1, 1, 24-40. https://doi.org/10.1016/j.eist.2011.02.002

Giddens, A. (1984): The Constitution of Society. Outline of the Theory of Structuration. Cambridge.

Greenwood, R.; Suddaby, R. (2006): Institutional Entrepreneurship In Mature Fields: The Big Five Accounting Firms. In: Academy of Management Journal 49, 1, 27-48. https://doi.org/10.2307/20159744

Grin, J.; Rotmans, J.; Schot, J.W. (2010): Transitions to Sustainable Development: New Directions in the Study of Long Term Transformative Change. New York.

Hajer, M.A. (1993): Discourse Coalitions and the Institutionalisation of Practice. The Case of Acid Rain in Great Britain. In: Fischer, F.; Forester, J. (Hrsg.): The Argumentative Turn in Policy Analysis and Planning. Durham, 43-67.

Hansen, T.; Coenen, L. (2015). The geography of sustainability transitions: Review, synthesis and reflections on 
an emergent research field. In: Environmental Innovation and Societal Transitions 17, 92-109. https://doi.org/10. 1016/j.eist.2014.11.001

Hodson, M.; Geels, F.W.; McMeekin, A. (2017): Reconfiguring Urban Sustainability Transitions, Analysing Multiplicity. In: Sustainability 9, 2, 299. https://doi.org/10. 3390/su9020299

Hodson, M.; Marvin, S. (2017): Intensifying or transforming sustainable cities? Fragmented logics of urban environmentalism. In: Local Environment 22, S1, 8-22. https://doi.org/10.1080/13549839.2017.1306498

Honey-Rosés, J.; Stevens, M. (2019): Commentary on the Absence of Experiments in Planning. In: Journal of Planning Education and Research 39, 3, 267-272. https://doi. org/10.1177/0739456X17739352

Kanning, H. (2018): Reallabore aus planerischer Perspektive. Hannover. = sustainify Arbeits- und Diskussionspapier 3.

Kemp, R.; Schot, J.; Hoogma, R. (1998): Regime Shifts to Sustainability Through Processes of Niche Formation: The Approach of Strategic Niche Management. In: Technology Analysis and Strategic Management 10, 2, 175-198. https://doi.org/10.1080/09537329808524310

Klein, C.; Martinez, M. (2009): Wirklichkeitserzählungen. Felder, Formen und Funktionen nicht-literarischen Erzählens. In: Klein, C.; Martinez, M. (Hrsg.): Wirklichkeitserzählungen. Felder, Formen und Funktionen nichtliterarischen Erzählens. Stuttgart, 1-13.

Lam, D.P.M.; Martín-López, B.; Wiek, A.; Bennett, E.M.; Frantzeskaki, N.; Horcea-Milcu, A.I.; Lang, D.J. (2020): Scaling the impact of sustainability initiatives: a typology of amplification processes. In: Urban Transformations 2, 3. https://doi.org/10.1186/s42854-020-00007-9

Lawrence, T.B.; Suddaby, R. (2006): Institutions and Institutional Work. In: Clegg, S.R.; Hardy, C.; Lawrence, T.B.; Nord, W.R. (Hrsg.): The SAGE Handbook of Organization Studies. London, 215-254. https://doi.org/10.4135/ 9781848608030.n7

Loorbach, D.; Wittmayer, J.; Avelino, F.; von Wirth, T.; Frantzeskaki, N. (2020): Transformative innovation and translocal diffusion. In: Environmental Innovation and Societal Transitions 35, 251-260. https://doi.org/10. 1016/j.eist.2020.01.009

Mayring, P. (2015): Qualitative Inhaltsanalyse: Grundlagen und Techniken. Weinheim.

McPhearson, T.; Wijsman, K. (2017): Transitioning Complex Urban Systems: The Importance of Urban Ecology for Sustainability in New York City. In: Frantzeskaki, N.; Castán Broto, V.; Coenen, L.; Loorbach, D. (Hrsg.): Urban Sustainability Transitions. New York, 65-85.

Nevens, F.; Frantzeskaki, N.; Gorissen, L.; Loorbach, D. (2013): Urban Transition Labs: co-creating transforma- tive action for sustainable cities. In: Journal of Cleaner Production 50, 111-122. https://doi.org/10.1016/j. jclepro.2012.12.001

Nowotny, H. (2003): Democratising expertise and socially robust knowledge. In: Science and Public Policy 30, 3, 151-156. https://doi.org/10.3152/147154303781780461

Pentland, B.T. (1999): Building Process Theory with Narrative: From Description to Explanation. In: Academy of Management Review 24, 4, 711-724. https://doi.org/10. 2307/259350

Prince, G. (1982): Narratology. The Form and Functioning of Narrative. Berlin.

Raven, R. (2005): Strategic Niche Management for Biomass: A Comparative Study on the Experimental Introduction of Bioenergy Technologies in the Netherlands and Denmark. Saarbrücken.

Raven, R.; Sengers, F.; Spaeth, P.; Xie, L.; Cheshmehzangi, A.; de Jong, M. (2019): Urban experimentation and institutional arrangements. In: European Planning Studies 27, 2, 258-281. https://doi.org/10.1080/09654313.2017. 1393047

Schäpke, N.; Stelzer, F.; Bergmann, M.; Singer-Brodowski, M.; Wanner, M.; Caniglia, G.; Lang, D.J. (2017): Reallabore im Kontext transformativer Forschung. Ansatzpunkte zur Konzeption und Einbettung in den internationalen Forschungsstand. Lüneburg. = IETSR Discussion Papers in Transdisciplinary Sustainability Research 1/2017.

Schäpke, N.; Stelzer, F.; Caniglia, G.; Bergmann, M.; Wanner, M.; Singer-Brodowski, M.; Loorbach, D.; Olsson, P.; Baedeker, C.; Lang, D.J. (2018). Jointly Experimenting for Transformation? Shaping Real-World Laboratories by Comparing Them. GAIA - Ecological Perspectives for Science and Society 27, S1, 85-96. https://doi.org/10. 14512/gaia.27.S1.16

Schneidewind, U.; Augenstein, K.; Stelzer, F.; Wanner, M. (2018): Structure Matters: Real-World Laboratories as a New Type of Large-Scale Research Infrastructure: A Framework Inspired by Giddens' Structuration Theory. In: GAIA - Ecological Perspectives for Science and Society 27, S1, 12-17. https://doi.org/10.14512/gaia.27.S1. 5

Schneidewind, U.; Scheck, H. (2013): Die Stadt als „Reallabor" für Systeminnovationen. In: Rückert-John, J. (Hrsg.): Soziale Innovation und Nachhaltigkeit. Wiesbaden, 229-248. https://doi.org/10.1007/978-3-53118974-1_12.

Scholes, R. (1981): Language, Narrative, and Anti-Narrative. In: Mitchell, W.J.T. (Hrsg.): On Narrative. Chicago, 200-208.

Schot, J.; Geels, F.W. (2008): Strategic niche management and sustainable innovation journeys: theory, findings, research agenda, and policy. In: Technology Analysis and 
Strategic Management 20, 5, 537-554. https://doi.org/ 10.1080/09537320802292651

Sengers, F.; Wieczorek, A.J.; Raven, R. (2019): Experimenting for sustainability transitions: A systematic literature review. In: Technological Forecasting and Social Change 145, 153-164. https://doi.org/10.1016/j.techfore.2016. 08.031

Sharp, D. (2018): Sharing Cities for Urban Transformation: Narrative, Policy and Practice. In: Urban Policy and Research 36, 4, 513-526. https://doi.org/10.1080/ 08111146.2017 .1421533

Somers, M.R.; Gibson, G.D. (1993): Reclaiming the Epistemological "Other": Narrative and the Social Constitution of Identity. Ann Arbor.

Thornton, P.H.; Ocasio, W. (1999): Institutional Logics and the Historical Contingency of Power in Organizations: Executive Succession in the Higher Education Publishing Industry, 1958-1990. In: American Journal of Sociology 105, 3, 801-843. https://doi.org/10.1086/210361

Thornton, P.H.; Ocasio, W. (2008): Institutional Logics. In: Greenwood, R.; Oliver, C.; Suddaby, R.; Sahlin, K. (Hrsg.): The SAGE Handbook of Organizational Institutionalism. London, 99-128. https://doi.org/10.4135/ 9781849200387.n4

Thornton, P.H.; Ocasio, W.; Lounsbury, M. (2012): The Institutional Logics Perspective: A New Approach to Culture, Structure and Process. Oxford. https://doi.org/10. 1093/acprof:oso/9780199601936.001.0001

Unruh, G.C. (2000). Understanding carbon lock-in. In:
Energy Policy 28, 12, 817-830. https://doi.org/10.1016/ S0301-4215(00)00070-7

von Wirth, T., Fuenfschilling, L.; Frantzeskaki, N.; Coenen, L. (2019): Impacts of urban living labs on sustainability transitions: mechanisms and strategies for systemic change through experimentation. In: European Planning Studies 27, 2, 229-257. https://doi.org/10.1080/ 09654313.2018.1504895

Wanner, M.; Hilger, A.; Westerkowski, J.; Rose, M.; Stelzer, F.; Schäpke, N. (2018): Towards a Cyclical Concept of Real-World Laboratories. A Transdisciplinary Research Practice for Sustainability Transitions. In: DisP - The Planning Review 54, 2, 94-114. https://doi.org/10.1080/ 02513625.2018.1487651

WBGU - Wissenschaftlicher Beirat der Bundesregierung Globale Umweltveränderungen (2016): Der Umzug der Menschheit. Die transformative Kraft der Städte. Berlin.

Wittmayer, J.M.; Backhaus, J.; Avelino, F.; Pel, B.; Strasser, T.; Kunze, I. (2015): Narratives of change: How Social Innovation Initiatives engage with their transformative ambitions. o.O. $=$ TRANSIT Working Paper 4.

Wolfram, M.; Frantzeskaki, N. (2016): Cities and Systemic Change for Sustainability: Prevailing Epistemologies and an Emerging Research Agenda. In: Sustainability 8, 2, 144. https://doi.org/10.3390/su8020144

Wolfram, M.; Frantzeskaki, N.; Maschmeyer, S. (2016): Cities, systems and sustainability: status and perspectives of research on urban transformations. In: Current Opinion in Environmental Sustainability 22, 18-25. https:// doi.org/10.1016/j.cosust.2017.01.014 